Kate A Curtis

$\mathrm{PhD}, \mathrm{RN}$

Postdoctoral Fellow

Rebecca J Mitchell MA (Psych), MOHS, PhD,

Research Fellow

Shanley S Chong

$\mathrm{PhD}$

Biostatistician

Zsolt J Balogh

MD, PhD, FRACS

Director of Trauma

Duncan J Reed

MB BS, FACEM

Director of Trauma

Peter T Clark

FACEM, FCICM, FANZCA

Clinical Director

Scott D'Amours

MDCM, FRCS(C), FRACS

Trauma Director

Deborah A Black

PhD, MStat, BSc,

Associate Dea

Staff Development

Mary E Langcake

BM BS, BSC(Hons), FRACS,

Iman B Taylo

MND,

Research Fellow

Patricia McDougal

RN, Cert Hlth Economics,

Executive

Peter A Cameron

MB BS, MD, FACEM

Head

1 Sydney Nursing School,

University of Sydney,

Sydney, NSW.

2 St George Hospital

Sydney, NSW.

3 Transport and Road

Safety Research, School of

Aviation, University of New

South Wales, Sydney, NSW.

4 Centre for Research,

Evidence Management and

Surveillance, Sydney South

West Local Health District,

New South Wales Health.

Sydney, NSW.

5 John Hunter Hospital,

University of Newcastle.

Newcastle, NSW.

6 Emergency Department,

Gosford Hospital,

Gosford, NSW.

7 New South Wales

Institute of Trauma and

Injury Management

Sydney, NSW.

8 Liverpool Hospital

Sydney, NSW.

9 Faculty of Health

Sciences,

University of Sydney,

Sydney, NSW.

10 The George Institute for Global Health

Sydney, NSW.

11 Critical Care Division School of Public Health,

Monash University,

Melbourne, VIC.

kate.curtis@

sydney.edu.au

MJA 2012; 197: 233-237 doi: $10.5694 /$ mjall.1135

\title{
Injury trends and mortality in adult patients with major trauma in New South Wales
}

1 raumatic injury accounts for $9 \%$ of global mortality, ${ }^{1}$ and imposes significant physical and psychological disability on people of all age groups. It remains the leading cause of death in those under 45 years of age. ${ }^{2}$ Trauma systems facilitate the timely treatment of patients with major trauma at a trauma centre. This approach has reduced mortality in patients with trauma in Australia ${ }^{3,4}$ and internationally. 5,6

A system for trauma care was first proposed in Australia in New South Wales in 1988 and then implemented in $1992 .{ }^{7}$ The paediatric trauma system in NSW has been studied previously, ${ }^{8}$ but the trauma system for the adult population has not been evaluated in NSW. A monitoring program was established in 2002 (the NSW Trauma Registry) by the NSW Institute of Trauma and Injury Management. A predefined, mandatory minimum dataset for patients with major injury (injury severity score [ISS] > 15) is collected by trained trauma nurses in each hospital and submitted electronically to the centrally maintained registry. Variables include 26 non-identifiable items about each injured person, including the time and mechanism of injury, the ISS, the prehospital transport mode and inhospital treatment.

Trauma centres in Australia are categorised according to model resource levels (summarised in Box 1). ${ }^{9}$ During the study period, patients with major trauma were transported to the nearest Level I centre, or to a regional trauma centre, commensurate with a Level III centre. Regional trauma centres play an important role in the delivery of trauma care, but often lack specialty resources. Most are located outside Sydney, and road travel time from regional trauma centres to a Level I centre ranges from 30 minutes to 2 hours. In a recent single region study in NSW, morbidity was significantly higher in a regional trauma centre compared with a Level I centre. ${ }^{10}$

Our aim was to examine trends and factors associated with mortality in

Objective: To examine trends in mechanism and outcome of major traumatic injury in adults since the implementation of the New South Wales trauma monitoring program, and to identify factors associated with mortality.

Design and setting: Retrospective review of NSW Trauma Registry data from 1 January 2003 to 31 December 2007, including patient demographics, year of injury, and level of trauma centre where definitive treatment was provided.

Participants: 9769 people aged $\geqslant 15$ years hospitalised for trauma, with an injury severity score (ISS) $>15$.

Main outcome measures: The NSW Trauma Registry outcome measures included were overall hospital length of stay, length of stay in an intensive care unit and inhospital mortality.

Results: There was a decreasing trend in severe trauma presentations in the age group 16-34 years, and an increasing trend in presentations of older people, particularly those aged $\geqslant 75$ years. Road trauma and falls were consistently the commonest injury mechanisms. There were 1328 inhospital deaths (13.6\%). Year of injury, level of trauma centre, ISS, head/neck injury and age were all independent predictors of mortality. The odds of mortality was significantly higher among patients receiving definitive care at regional trauma centres compared with Level I centres (odds ratio, 1.34; 95\% Cl, 1.10-1.63).

Conclusions: Deaths from major trauma in NSW trauma centres have declined since 2003, and definitive care at a Level 1 trauma centre was associated with a survival benefit. More comprehensive trauma data collection with timely analysis will improve injury surveillance and better inform health policy in NSW.

adult patients with major traumatic injury in NSW.

\section{Methods}

A retrospective review of patient demographics, injury type, transport mode, mechanism and outcomes was conducted using data from the NSW Trauma Registry, 1 January 2003 - 31 December 2007. Ethics approval for the study was obtained from the St George Hospital Human Research Ethics Committee (HREC 08/STG/166).

Patients aged $\geqslant 15$ years with major injuries, defined as an ISS $>15$, were included. The ISS was calculated from the Abbreviated Injury Scale, 1998 revision. ${ }^{11}$
There were no changes in trauma centre designation or data collection resources during the study period.

Eight adult Level I centres and three regional trauma centres in the Greater Sydney Metropolitan region (Sydney metropolitan region, including the Illawarra, Hawkesbury-Nepean and Central Coast regions) submitted data to the NSW Trauma Registry. There were no designated rural trauma services during the study period.

Definitive trauma care was considered to be delivered at the hospital where most of the patient's treatment and care were provided, as defined by length of stay (LOS). Outcome measures were limited to those included in

\section{Trauma service levels}

Level I/II: 24-hour full spectrum of care for the most critically injured patients - reception, resuscitation, and rehabilitation and, ideally, a surgical trauma admitting service. Only Level I includes research, education and fellowship training, quality improvement, prevention and outreach programs

Level III or regional trauma centre: prompt assessment, resuscitation, 24-hour on-call emergency and general surgical and anaesthetic services. Provides stabilisation for patients with major trauma while arranging transfer to Level I/II, or definitive care according to patient needs and available resources

Level IV: hospital with resuscitation facilities and medical attention available within 30 minutes. Patients with major trauma are transferred as soon as possible

Level V: established tertiary institutions not designated for trauma care; or, in rural areas, small hospitals or medical centres, with no immediately available medical practitioner

Adapted from the Australasian Trauma Verification Program Manual 2009.9 
2 Demographic and injury profile of 9769 patients with major trauma (injury severity score [ISS], > 15) in New South Wales, 2003-2007

\begin{tabular}{|c|c|c|c|c|c|c|c|}
\hline & 2003 & 2004 & 2005 & 2006 & 2007 & Total & $\chi^{2}$ test for trend \\
\hline NSW population $\geqslant 15$ years & 5378000 & 5438000 & 5508000 & 5574000 & 5640000 & & \\
\hline Patients $\geqslant 15$ years with ISS $>15$ & 1802 & 1924 & 1993 & 2089 & 1961 & 9769 & \\
\hline Mortality, $n$ (\%) & $272(15.1 \%)$ & $238(12.4 \%)$ & $290(14.6 \%)$ & $275(13.2 \%)$ & $253(12.9 \%)$ & 1328 & $P=0.07$ \\
\hline \multicolumn{8}{|l|}{ Sex*,n (\%) } \\
\hline Male & $1322(73.4 \%)$ & $1427(74.2 \%)$ & $1406(70.5 \%)$ & $1557(74.5 \%)$ & $1428(72.8 \%)$ & 7140 & $P=0.43$ \\
\hline Female & $479(26.6 \%)$ & $497(25.8 \%)$ & $587(29.5 \%)$ & $532(25.5 \%)$ & $532(27.1 \%)$ & 2627 & \\
\hline \multicolumn{8}{|l|}{ Age group, $n(\%)$} \\
\hline $16-24$ & $411(22.8 \%)$ & $374(19.4 \%)$ & $381(19.1 \%)$ & $392(18.8 \%)$ & $315(16.1 \%)$ & 1873 & $P<0.01$ \\
\hline $25-34$ & $288(16.0 \%)$ & $336(17.5 \%)$ & $322(16.2 \%)$ & $345(16.5 \%)$ & $310(15.8 \%)$ & 1601 & $P=0.51$ \\
\hline $35-44$ & $283(15.7 \%)$ & $275(14.3 \%)$ & $273(13.7 \%)$ & $275(13.2 \%)$ & $286(14.6 \%)$ & 1392 & $P=0.10$ \\
\hline $45-54$ & $224(12.4 \%)$ & $239(12.4 \%)$ & $217(10.9 \%)$ & $237(11.3 \%)$ & $225(11.5 \%)$ & 1142 & $P=0.10$ \\
\hline $55-64$ & $164(9.1 \%)$ & $215(11.2 \%)$ & $204(10.2 \%)$ & $239(11.4 \%)$ & $201(10.2 \%)$ & 1023 & $P=0.13$ \\
\hline $65-74$ & $164(9.1 \%)$ & $164(8.5 \%)$ & $190(9.5 \%)$ & $200(9.6 \%)$ & $193(9.8 \%)$ & 911 & $P=0.11$ \\
\hline $75-84$ & $180(10.0 \%)$ & $232(12.1 \%)$ & $278(13.9 \%)$ & $254(12.2 \%)$ & $270(13.8 \%)$ & 1214 & $P<0.01$ \\
\hline $85+$ & $88(4.9 \%)$ & $89(4.6 \%)$ & $128(6.4 \%)$ & $147(7.0 \%)$ & $161(8.2 \%)$ & 613 & $P<0.01$ \\
\hline \multicolumn{8}{|l|}{ Injury severity score (ISS), $n$ (\%) } \\
\hline Median ISS & 22 & 22 & 22 & 22 & 22 & 22 & \\
\hline Serious (ISS, 16-24) & $1006(55.8 \%)$ & $1103(57.3 \%)$ & $1110(55.7 \%)$ & $1150(55.1 \%)$ & $1123(57.3 \%)$ & 5492 & $P=0.50$ \\
\hline Severe (ISS, 25-39) & $648(36.0 \%)$ & $670(34.8 \%)$ & 717 (36.0\%) & 770 (36.9\%) & 711 (36.3\%) & 3516 & $P=0.76$ \\
\hline Critical (ISS, 40-75) & $148(8.2 \%)$ & $151(7.8 \%)$ & $166(8.3 \%)$ & $169(8.1 \%)$ & $127(6.5 \%)$ & 761 & $P=0.18$ \\
\hline \multicolumn{8}{|l|}{ Injury mechanism, $n$ (\%) } \\
\hline Burns & $38(2.1 \%)$ & $45(2.3 \%)$ & $29(1.5 \%)$ & $29(1.4 \%)$ & $26(1.3 \%)$ & 167 & $P<0.01$ \\
\hline Falls & $585(32.5 \%)$ & $654(34.0 \%)$ & $700(35.1 \%)$ & $758(36.3 \%)$ & 778 (39.7\%) & 3475 & $P<0.01$ \\
\hline Road trauma & $804(44.6 \%)$ & $872(45.3 \%)$ & $907(45.5 \%)$ & $920(44.0 \%)$ & 774 (39.5\%) & 4277 & $P=0.11$ \\
\hline Motor vehicle & $424(23.5 \%)$ & $469(24.4 \%)$ & $474(23.8 \%)$ & $462(22.1 \%)$ & $375(19.1 \%)$ & 2204 & $P<0.01$ \\
\hline Motorcycle & $175(9.7 \%)$ & $184(9.6 \%)$ & $207(10.4 \%)$ & $215(10.3 \%)$ & $185(9.4 \%)$ & 966 & $P=0.47$ \\
\hline Pedestrian & $163(9.0 \%)$ & $173(9.0 \%)$ & $183(9.2 \%)$ & $183(8.8 \%)$ & $155(7.9 \%)$ & 857 & $P=0.11$ \\
\hline Pedal cyclist & $42(2.3 \%)$ & $46(2.4 \%)$ & $43(2.2 \%)$ & $60(2.9 \%)$ & $59(3.0 \%)$ & 250 & $P=0.05$ \\
\hline Recreation & $68(3.8 \%)$ & $46(2.4 \%)$ & $46(2.3 \%)$ & $69(3.3 \%)$ & $53(2.7 \%)$ & 282 & $P=0.18$ \\
\hline Violence (assault, stabbing, gunshot wound, self-harm) & $197(10.9 \%)$ & $208(10.8 \%)$ & $204(10.2 \%)$ & $220(10.5 \%)$ & $223(11.4 \%)$ & 1052 & $P=0.39$ \\
\hline Other/unknown & $110(6.1 \%)$ & $99(5.1 \%)$ & $107(5.4 \%)$ & $93(4.4 \%)$ & $107(5.5 \%)$ & 516 & $P=0.08$ \\
\hline
\end{tabular}

the NSW Trauma Registry: overall hospital LOS, LOS in an intensive care unit (ICU) and inhospital mortality. Injury severity was grouped into serious (ISS, 16-24), severe (ISS, 2539) and critical (ISS, 40-75). ${ }^{4}$

\section{Data analysis}

Analysis was performed using SAS 9.1 for Windows (SAS Institute Inc, Cary, NC, USA). Multivariate logistic regression was used to assess the association between inhospital mortality, location of definitive care and year. Adjusted odds ratios (ORs) and 95\% CIs for each year, relative to 2003, were calculated. Sex, age, ISS, body region affected and initial treatment location were included in all models as covariates. Interactions between relevant explanatory variables were examined. First-order interactions only and $P$ values $<0.05$ were considered significant. All models were assessed by the Hosmer-Lemeshow goodness-of-fit test calibration $\left(\chi^{2}=\right.$ 13.45; $\mathrm{df}=8 ; P=0.10) \cdot \chi^{2}$ tests for trend were used to compare categorical outcome variables.

\section{Results}

During the 5-year period, 9769 people aged $\geqslant 15$ years and admitted to hospital with an ISS $>15$ had their details recorded in the NSW Trauma Registry. The patients' mean age was 48.2 years $(\mathrm{SD}, 22.6)$ and $73 \%$ were male. There was a trend towards increasing presentations of patients aged 75 years and over $\left(\chi^{2}\right.$ test for trend, $\left.P<0.01\right)$. The median ISS remained consistent at 22 over the 5 -year period (Box 2).

Road trauma and falls were the most common mechanisms of injury, with motor vehicle accidents reducing as a cause of injury, and falls of $<1 \mathrm{~m}$ increasing as a cause $(P<0.01)$. The mode of transport from the injury scene remained consistent, with 79\% arriving by road ambulance, $12 \%$ by helicopter and the remaining $9 \%$ by private transport.

\section{Treatment location, transfer and length of hospital stay}

Since 2003, there has been a significant increase in the number of patients receiving initial treatment at regional trauma centres $(P=0.01)$, corresponding to a slight decrease in the number of patients receiving initial treatment in the Sydney metropolitan region $(P=0.03$; OR, 0.66 ; 95\% CI, 0.54-0.81).

The number of interhospital transfers between regional trauma centres and Level I centres did not increase significantly during the 5 -year period (range, $71-79 ; P=0.46$ ). The mortality 
3 Independent predictors of inhospital mortality in patients with major trauma head and neck injuries, road trauma, falls and injuries caused by violence, New South Wales, 2003-2007

Odds ratios of inhospital mortality $(95 \% \mathrm{Cl})$

\begin{tabular}{|c|c|c|c|c|c|}
\hline & & & & & \\
\hline & All patients & Head and neck injuries & Road trauma & Falls & Violence \\
\hline \multicolumn{6}{|l|}{ Year } \\
\hline 2003 [Reference] & 1 & 1 & 1 & 1 & 1 \\
\hline 2004 & $0.74(0.60-0.91)$ & $0.72(0.57-0.92)$ & $0.77(0.54-1.10)$ & $0.77(0.56-1.06)$ & $0.48(0.22-1.06)$ \\
\hline 2005 & $0.84(0.69-1.03)$ & $0.88(0.70-1.10)$ & $0.88(0.64-1.22)$ & 0.79 (0.57-1.06) & $0.77(0.37-1.61)$ \\
\hline 2006 & $0.72(0.59-0.88)$ & $0.71(0.57-0.90)$ & $0.68(0.49-0.96)$ & $0.67(0.49-0.91)$ & $1.18(0.60-2.32)$ \\
\hline 2007 & $0.71(0.58-0.88)$ & $0.68(0.54-0.86)$ & $0.72(0.50-1.03)$ & $0.62(0.45-0.85)$ & $0.80(0.38-1.66)$ \\
\hline \multicolumn{6}{|l|}{ Sex } \\
\hline Male & 1.01 (0.87-1.16) & 1.01 (0.86-1.19) & $1.21(0.95-1.56)$ & 0.95 (0.77-1.16) & $0.61(0.32-1.17)$ \\
\hline \multicolumn{6}{|l|}{ Age group } \\
\hline 16-24 [Reference] & 1 & 1 & 1 & 1 & 1 \\
\hline $25-34$ & 1.33 (1.03-1.71) & 1.46 (1.09-1.97) & $1.25(0.89-1.75)$ & $0.89(0.43-1.86)$ & $1.63(0.83-3.21)$ \\
\hline $35-44$ & $1.34(1.03-1.75)$ & $1.55(1.14-2.11)$ & $1.15(0.79-1.68)$ & $1.34(0.69-2.62)$ & $2.04(1.02-4.09)$ \\
\hline $45-54$ & 1.41 (1.07-1.87) & $1.55(1.12-2.14)$ & $1.18(0.79-1.76)$ & $1.36(0.71-2.61)$ & $2.50(1.10-5.71)$ \\
\hline $55-64$ & $1.65(1.24-2.19)$ & $1.77(1.28-2.44)$ & $1.51(0.98-2.35)$ & $1.47(0.79-2.73)$ & $1.53(0.54-4.29)$ \\
\hline $65-74$ & 3.35 (2.57-4.38) & $3.64(2.70-4.91)$ & $4.26(2.83-6.41)$ & $2.72(1.51-4.92)$ & $4.78(1.49-15.32)$ \\
\hline $75-84$ & $4.95(3.88-6.32)$ & $5.17(3.93-6.80)$ & $4.00(2.66-6.01)$ & $4.53(2.57-7.99)$ & $6.28(1.60-24.69)$ \\
\hline $85+$ & $9.01(6.85-11.87)$ & $8.44(6.23-11.45)$ & $21.21(11.82-38.05)$ & $6.37(3.56-11.40)$ & $29.87(3.05-292.75)$ \\
\hline \multicolumn{6}{|l|}{ Hospital level for definitive care } \\
\hline Level I [Reference] & 1 & 1 & 1 & 1 & 1 \\
\hline Regional trauma centre & $1.34(1.10-1.63)$ & $1.20(0.99-1.56)$ & $1.08(0.78-1.49)$ & $1.42(1.03-1.95)$ & $1.24(0.58-2.65)$ \\
\hline \multicolumn{6}{|l|}{ Injury severity score (ISS) } \\
\hline Serious (ISS, 16-24) [Reference] & 1 & 1 & 1 & 1 & 1 \\
\hline Severe (ISS, 25-39) & $6.33(5.38-7.44)$ & $6.59(5.48-7.92)$ & $9.88(7.02-13.92)$ & $4.79(3.86-5.95)$ & $8.69(4.95-15.25)$ \\
\hline Critical (ISS, 40-75) & $30.42(24.60-37.60)$ & $26.05(20.34-33.36)$ & 49.02 (34.05-70.59) & $25.16(16.19-39.09)$ & 83.09 (32.11-214.99) \\
\hline \multicolumn{6}{|l|}{ Body region } \\
\hline Head or neck [Reference] & 1 & - & 1 & 1 & 1 \\
\hline Face & $0.37(0.17-0.81)$ & - & $0.37(0.13-1.07)$ & $0.66(0.19-2.30)$ & 1 \\
\hline Chest & $0.79(0.65-0.96)$ & - & $0.79(0.60-1.05)$ & $0.49(0.31-0.76)$ & $1.42(0.80-2.51)$ \\
\hline Abdominal or pelvic contents & $0.67(0.48-0.94)$ & - & $0.63(0.39-1.01)$ & $0.96(0.49-1.90)$ & $0.91(0.34-2.46)$ \\
\hline Extremities or pelvic girdle & $0.61(0.34-1.12)$ & - & $0.98(0.47-2.04)$ & $0.38(0.10-1.41)$ & $1.31(0.21-8.27)$ \\
\hline External/burns & $2.42(1.61-3.66)$ & - & $3.67(0.74-18.20)$ & - & - \\
\hline $\begin{array}{l}\text { Hosmer-Lemeshow goodness- } \\
\text { of-fit calibration statistic }\end{array}$ & $\begin{array}{c}\chi^{2}=15.24 ; \mathrm{df}=8 \\
P=0.05\end{array}$ & $\begin{array}{c}\chi^{2}=13.45 ; \mathrm{df}=8 \\
P=0.10\end{array}$ & $\begin{array}{c}\chi^{2}=7.10 ; d f=8 \\
P=0.53\end{array}$ & $\begin{array}{c}\chi^{2}=6.20 ; d f=8 \\
P=0.63\end{array}$ & $\begin{array}{c}\chi^{2}=14.13 ; \mathrm{df}=8 \\
\quad P=0.08\end{array}$ \\
\hline
\end{tabular}

rate for those patients transferred $(n=37)$ halved over this time $(16.9 \%$ to $7.6 \%)(P=0.06)$. There was a nonsignificant reduction in the proportion of patients receiving initial treatment in a rural or remote area, from $5.0 \%$ to $3.7 \%(P=0.2)$. There was a significant decrease in the proportion of patients admitted to an ICU $(P<0.01)$, although the overall number of patients admitted $(n=880)$ and the LOS in ICU (median, 4 days) remained steady. Total bed-days during the study period were 163810 . The median LOS reduced slightly from 10 to 9 days. There was no significant difference in LOS by level of trauma centre.

\section{Mortality}

There were 1328 inhospital deaths (overall mortality rate, $13.6 \%$; range, $15 \%$ in 2002 to $12.9 \%$ in 2007). Year of injury, level of trauma centre, ISS, body region (Abbreviated Injury Scale) and age were independent predictors of mortality (Box 3). There was a significant decrease in the unadjusted mortality and the number of patients dying at Level I centres over time $(P=0.02)$. The adjusted relative odds of mortality decreased significantly from 2003 to 2007, except in 2005 (Box 4). The relative odds of mortality were significantly higher among patients receiving definitive care at regional trauma centres (OR,
1.34; 95\% CI, 1.10-1.63) compared with Level I centres.

There was no significant difference in patient characteristics between patients who died in a regional trauma centre versus a Level I centre, except for mechanism of injury $\left(\chi^{2}=20.15 ; \mathrm{df}=5 ; P<0.01\right)$. Level I centres received a higher proportion of fatally injured patients who were pedestrians, had fallen $>1 \mathrm{~m}$, or suffered burns or violence (eg, assault, stabbing). Deaths at regional trauma centres more commonly resulted from motorcycle or pedal cycle crashes.

The relative odds of death increased in those older than 65 years, and increased 30-fold in those with critical 


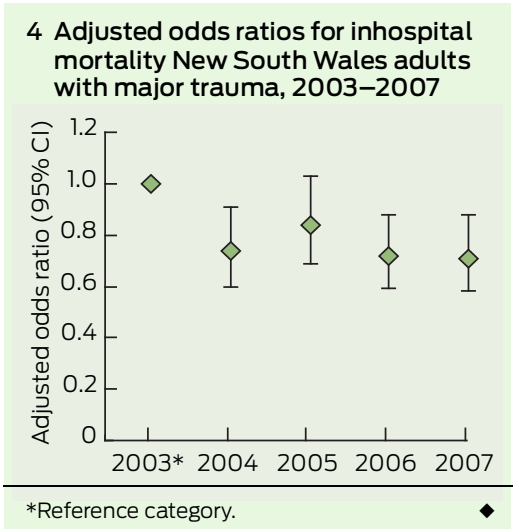

injuries (ISS, 40-75). In patients with head and neck injuries, the relative odds of death decreased significantly over time to 2007 (OR, 0.68; 95\% CI, $0.54-0.86)$, particularly in older $(>65$ years) and more severely injured patients (Box 3 ).

\section{Discussion}

This is the first statewide analysis of major adult trauma trends and outcomes in NSW. Age, sex, injury severity and initial treatment at a regional trauma centre were independent predictors of mortality. Despite a small reduction in mortality after major trauma over the study period, trauma remains a significant cause of mortality and hospitalisation in NSW, and accurate data on trauma-related mortality are, at present, not recorded for rural areas. Trauma should continue to feature prominently on the public health agenda. ${ }^{12}$

The overall mortality rate in NSW is similar to that reported in other Australian states. ${ }^{4,13}$ During the study period no change in prehospital protocol occurred, yet the proportion of patients receiving care at a regional trauma centre increased. This may represent improved data collection and reporting at these centres, as trauma coordinators became more experienced.

Patients admitted to a regional trauma centre had a significantly higher mortality rate compared with those admitted to a Level I centre. The survival benefit at Level I centres could be explained by the presence of greater resources, such as the availability of surgically trained staff 24 hours a day, a multidisciplinary trauma service ${ }^{14,15}$ and higher patient volumes, resulting in enhanced expertise in trauma care. ${ }^{16}$ Deficiencies in trauma care, regardless of trauma centre level, may occur at multiple points of care and are more likely to occur when there is failure to comply with protocols, poor communication and/or delayed referral. ${ }^{10,14,17,18}$ However, without reviewing each of the deaths in this study, it is not possible to comment on factors contributing to mortality.

The benefits of primary transport or early interhospital transfer of patients with major trauma to a Level I trauma centre are confirmed in our study. In 2008 Ambulance NSW introduced "Protocol T1", which specifies that patients with major trauma should be taken directly to a Level I centre, bypassing lower-level centres, if transport time is within 1 hour. ${ }^{19}$ The impact of Protocol T1 on patient transport practice and outcomes requires evaluation.

The geography of NSW means that regional trauma centres will continue to play an important role in initial stabilisation of patients with trauma. Our study suggests that improved adherence to transfer guidelines will contribute to improved patient outcomes consistent with the experience in Victoria ${ }^{17}$ and the United States. ${ }^{20}$ In recent years, a series of standardised transfer guidelines for patients with major trauma have been implemented in NSW, with variable compliance. ${ }^{10,21}$ The low numbers of transferred patients could also result from some patients with critical injuries being deemed too unstable for transfer, transfer being considered futile, or patients dying before a transfer decision was made. ${ }^{10,21}$

Our study has several limitations. No validation checks were conducted on the trauma data (ISS and body region) because Abbreviated Injury Scale scores were not recorded. Outcome analysis and conclusions are limited by lack of data describing patients' physiological status, comorbidities and prehospital transport time. There is potential selection bias because some patients may have been too unstable for transfer or died before transfer. In addition, some patients with an ISS $>15$ are managed initially at smaller rural or regional hospitals, many of which are not designated or resourced to submit data to the NSW Trauma Registry. However, since the study period, seven rural and regional trauma services have been allocated funding for trauma nurse consultants and data managers. One burns referral centre does not contribute to the NSW Trauma Registry.

All the above factors contribute to underreporting of the incidence of major injury in adults. The linkage of data on deaths from the National Coroners Information System and ambulance records to trauma data would provide more accurate information to better inform injury prevention strategies, research priorities and trauma system design and planning.

Trauma systems in Australia have been developed largely on the basis of expert opinion, international experience and informal consensus. Each trauma system needs to consider the specific needs of the region, and the available resources and expertise. ${ }^{15}$ It is imperative to continue to evaluate trauma systems, and central to this is quality surveillance data. ${ }^{22}$ In 2010, a statewide online trauma registry was established to facilitate data collection; however, the amount of data each trauma centre collects and enters remains variable. The resourcing of an enhanced statewide minimum dataset, including physiological (vital signs) function and data on cause and time of death, would enable closer analysis of the NSW trauma system. However, ongoing independent academic support is required to conduct, interpret and translate the results of a sustained trauma monitoring program. This would enable targeted and evidence-based resource allocation for initiatives such as increasing helicopter primary retrieval capability from regional areas, or improving education and training for staff in regional trauma centres.

Mortality and LOS are crude methods of appraising outcome, and do not reflect the full burden of injury, although they allow simple monitoring of trauma systems. NSW Level I trauma centres appear to have been effective in reducing mortality. This is likely to be a result of the development and uptake of evidence-based practice, including the NSW Ministry 
of Health clinical practice guidelines. $^{23}$ Where deficiencies are detected it is incumbent on governing authorities to ensure that all trauma centres are adequately resourced, staff are well trained, and compliance with trauma protocols are maintained. ${ }^{18}$ We recommend that the NSW Ministry of Health considers supporting a mandatory trauma verification process, such as that conducted by the Royal Australasian College of Surgeons. ${ }^{9}$ Currently, the government designates major trauma centres independently of trauma centre verification processes and standards. ${ }^{9}$

\section{Conclusions}

There appears to be a survival benefit associated with definitive care at a Level I trauma centre. More comprehensive trauma data collection with timely analysis will better inform evidence-based health policy in NSW. This can only occur with adequate resourcing. A mandatory trauma verification process would facilitate minimum standards of care.

Acknowledgements: We thank the trauma nurses and data managers from participating trauma centres in NSW and David Martens from the NSW Institute of Trauma and Injury Management for assistance with data provision. Rebecca Mitchell was supported by an Australian Research Council linkage postdoctoral fellowship (LP0990057). Data analysis was funded by the St George Honda Trauma and Critical Care Research Program.

Competing interests: No relevant disclosures.

Received 21 Oct 2011, accepted 13 Mar 2012.

1 Peden MM, McGee K, Krug E. Injury: a leading cause of the global burden of disease, 2000. Geneva:WHO, 2002
2 Australian Bureau of Statistics. Causes of Death, Australia, 2008. Canberra: ABS, 2010. (ABS Cat. No. 3303.0.) http://www.abs.gov.au/AUSSTATS/ abs@.nsf/Lookup/3303.0Main+Features12008? OpenDocument (accessed Mar 2012).

3 Brennan PW, Everest ER, Griggs WM, et al. Risk of death among cases attending South Australian major trauma services after severe trauma: the first 4 years of operation of a state trauma system. J Trauma 2002; 53: 333-339.

4 Cameron PA, Gabbe BJ, Cooper DJ, et al. A statewide system of trauma care in Victoria: effect on patient survival. Med J Aust 2008; 189: 546-550.

5 Celso B, Tepas J, Langland-Orban B, et al. A systematic review and meta-analysis comparing outcome of severely injured patients treated in trauma centers following the establishment of trauma systems. J Trauma 2006; 60: 371-378.

6 Twijnstra MJ, Moons KG, Simmermacher RK, Leenen LP. Regional trauma system reduces mortality and changes admission rates: a before and after study. Ann Surg 2010; 251: 339-343.

7 New South Wales Institute of Trauma and Injury Management. The NSW Trauma Registry profile of serious to critical injuries. Sydney: NSW Health, 2007. http://www.health.nsw.gov.au/pubs/ 2008/pdf/trauma_profile_2006.pdf (accessed Mar 2012).

8 Mitchell RJ, Curtis K, Chong S, et al. Comparative analysis of trends in paediatric trauma outcomes in New South Wales, Australia. Injury 2011; Dec 5 [Epub ahead of print].

9 Royal Australasian College of Surgeons. The Australasian Trauma Verification Program Manual. Melbourne: RACS, 2009. http:// www.surgeons.org/media/4940/2009-08-04 Trauma_Verification_Manual.pdf (accessed Ma 2012).

10 Curtis K, Chong S, Mitchell R, et al. Outcomes of severely injured adult trauma patients in an Australian health service: does trauma center level make a difference? World J Surg 2011; 35: 2332-2340.

11 Association for the Advancement of Automotive Medicine. The Abbreviated Injury Scale. 1998 Revision. Barrington, IL: AAAM, 1998.

12 National Public Health Partnership. The National Injury Prevention and Safety Promotion Plan: 2004-2014. Canberra: NPHP, 2005. http://www. nphp.gov.au/publications/sipp/nipspp.pdf (accessed Mar 2012).
13 Royal Perth Hospital Trauma Services. Annual Report - 2008. Perth: Royal Perth Hospital, 2008. http://www.rph.wa.gov.au/pdf/Trauma_ Report_2008.pdf (accessed Mar 2012).

14 Ursic C, Curtis K, Zou Y, Black D. Improved trauma patient outcomes after implementation of a dedicated trauma admitting service. Injury 2009; 40: 99-103.

15 Liberman M, Mulder DS, Jurkovich GJ, Sampalis JS. The association between trauma system and trauma center components and outcome in a mature regionalized trauma system. Surgery 2005; 137: 647-658.

16 Garwe T, Cowan LD, Neas BR, et al. Directness of transport of major trauma patients to a level I trauma center: a propensity-adjusted survival analysis of the impact on short-term mortality. J Trauma 2011; 70: 1118-1127.

17 McDermott FT, Cordner SM, Tremayne AB, et al. A "before and after" assessment of the influence of the new Victorian trauma care system (19971998 vs 2001-2003) on the emergency and clinical management of road traffic fatalities in Victoria. Melbourne: Consultative Committee on Road Traffic Fatalities in Victoria, 2003.

18 Chua WC, D'Amours SK, Sugrue M, et al. Performance and consistency of care in admitted trauma patients: our next great opportunity in trauma care? ANZ J Surg 2009; 79: 443-448.

19 Ambulance Service of New South Wales. Prehospital Management of Major Trauma. Protocol Tl. Trauma Triage Tool. Major Trauma Criteria (MIST). Rozelle: Ambulance Service of New South Wales, 2008.

20 Newgard CD, McConnell KJ, Hedges JR, Mullins RJ. The benefit of higher level of care transfer of injured patients from nontertiary hospital emergency departments. J Trauma 2007; 63: 965-971.

21 Smith J, Lynch J, Sugrue M, et al. An evaluation of compliance with practice guidelines on interhospital trauma transfer. Injury 2005; 36 1051-1057.

22 Mitchell RJ, McClure RJ, Williamson AM, McKenzie K. Implementing the national priorities for injury surveillance. Med J Aust 2008; 188: 405-408.

23 NSW Institute of Trauma and Injury Management. Trauma guidelines [various publications]. Sydney: NSW ITIM, 2007. http:// www.itim.nsw.gov.au/wiki/Trauma_guidelines_ section (accessed Feb 2012). 